\title{
Present Tense: A Peculiar Case of Pemphigus Vulgaris Presenting with Tense Blisters during the COVID-19 Pandemic
}

\author{
Dianne Katherine R. Salazar-Paras, MD and Mae N. Ramirez-Quizon, MD \\ Department of Dermatology, Philippine General Hospital, University of the Philippines Manila
}

\begin{abstract}
Pemphigus vulgaris is an autoimmune bullous dermatosis presenting with flaccid blisters and erosions. The morphology of pemphigus reflects the more superficial intraepidermal level of split seen histologically in contrast with pemphigoid, where the level of split is deep below the epidermis. This is a case of a 58-year-old male clinically presenting with arcuate tense bullae, which are more characteristic of the pemphigoid group of disorders, which revealed an intraepidermal split and tombstoning pattern of the basal epidermis on histopathology. Direct immunofluorescence revealed intercellular IgG and C3 distribution. Although this patient presented clinically with tense bullae, the histopathology and direct immunofluorescence results were consistent with pemphigus vulgaris.
\end{abstract}

Key Words: pemphigus vulgaris, tense blisters, direct immunofluorescence

\section{INTRODUCTION}

Pemphigus and pemphigoid are vesicobullous skin disorders that are initially differentiated by the characteristics of their respective blisters. That is, pemphigus presents with flaccid bullae, and pemphigoid presents with tense bullae. This dichotomous distinction between pemphigus and pemphigoid is a reflection of the known pathophysiology and level of split in these conditions - pemphigus is flaccid because the primary pathology is intraepidermal, and pemphigoid is tense because the primary pathology is subepidermal. ${ }^{1}$ This report presents a peculiar case of pemphigus vulgaris $(\mathrm{PV})$ in a 58-year old Filipino male who presented with tense vesicles and bullae in an annular configuration just before the outbreak of COVID-19 in the Philippines, which hindered him from seeking prompt medical attention from a specialist.

\section{CASE DESCRIPTION}

This was a case of a 58-year-old Filipino male known to have hypertension and heart failure of one-year duration, maintained on enalapril, digoxin, clopidogrel, spironolactone, and atorvastatin but with poor compliance due to financial constraints. He presented with a four-month history of multiple pruritic, tense vesicles and bullae on an erythematous base which started on both wrists with subsequent involvement of both lower legs and feet, thighs, forearms, trunk, and face with no associated mucosal involvement. Progression of skin lesions prompted him to consult a local physician who treated him as a case of bacterial infection for which he was prescribed different combinations of oral

Corresponding author: Dianne Katherine R. Salazar-Paras, MD Department of Dermatology Philippine General Hospital University of the Philippines Manila

Taft Avenue, Ermita, Manila 1000, Philippines

Email:drsalazarparas@up.edu.ph 
antibiotics and topical steroids, but none afforded relief of symptoms and clearance of the lesions. The patient did not observe any relationship between the appearance of new lesions and intake of gluten-rich foods. No other members of his family presented with a similar skin condition.

Unable to seek specialized care due to the COVID-19 lockdown, he resorted to wound care using a concoction of boiled guava leaves until his lesions became intensely painful. His neighbor referred him to our institution through telemedicine.

Upon evaluation of the photos sent through a smartphone, the lesions were composed of multiple tense vesicles and bullae, and erythematous round plaques, some eroded and some already crusted, in annular configuration distributed on the back, chest, abdomen, forearms, and lower extremities (Figures 1B, 1C, 1D, 1E, 1F). No mucosal lesions were appreciated (Figure 1A).

His Dermatological Quality of Life Index (DLQI), a validated self-administered measure of a dermatologic patient's quality of life, was 25 (dermatosis resulting in an extremely adverse effect on the patient's life) at this time. ${ }^{2}$ He repeatedly expressed how he already wished for death due to lack of sleep and anxiety brought about by his skin condition.

After the initial telemedicine consult, the patient's presentation was attributed either to bullous pemphigoid (BP) or linear IgA bullous dermatosis (LABD) as both would present with tense vesicles and bullae with no temporal association with intake of gluten-containing foods. However, because these tense blisters were in annular configuration, LABD was considered more likely. Laboratories were requested, including glucose-6-phosphate dehydrogenase (G6PD) assay in preparation for dapsone therapy, which is an effective treatment for LABD. ${ }^{3}$ While waiting for the results, the patient was initially treated with prednisone $0.5 \mathrm{mg} / \mathrm{kg} / \mathrm{day}$, a potent topical corticosteroid with antibiotics (betamethasone dipropionate + mupirocin), saline compress, and liberal emollients.

More than a week after initiation of therapy marked improvement and relief of pain and pruritus were reported (Figures 2A, 2B). However, new tense bullae still appeared on his lower extremities (Figure 2C).

Because mobility was strictly limited due to the enhanced community quarantine (ECQ) and lack of funds on the part of the patient, one of the authors traveled about 150 kilometers to see the patient in his home and obtained specimens for 4-mm skin punch biopsy and direct immunofluorescence studies. The necessary permits so the patient can travel to a nearby province where G6PD assay was available were still being processed.

Hematoxylin and eosin staining of the specimen from perilesional skin showed intraepithelial splitting leaving a single layer of keratinocytes attached to the basement membrane resembling a row of tombstones. In the epidermis,
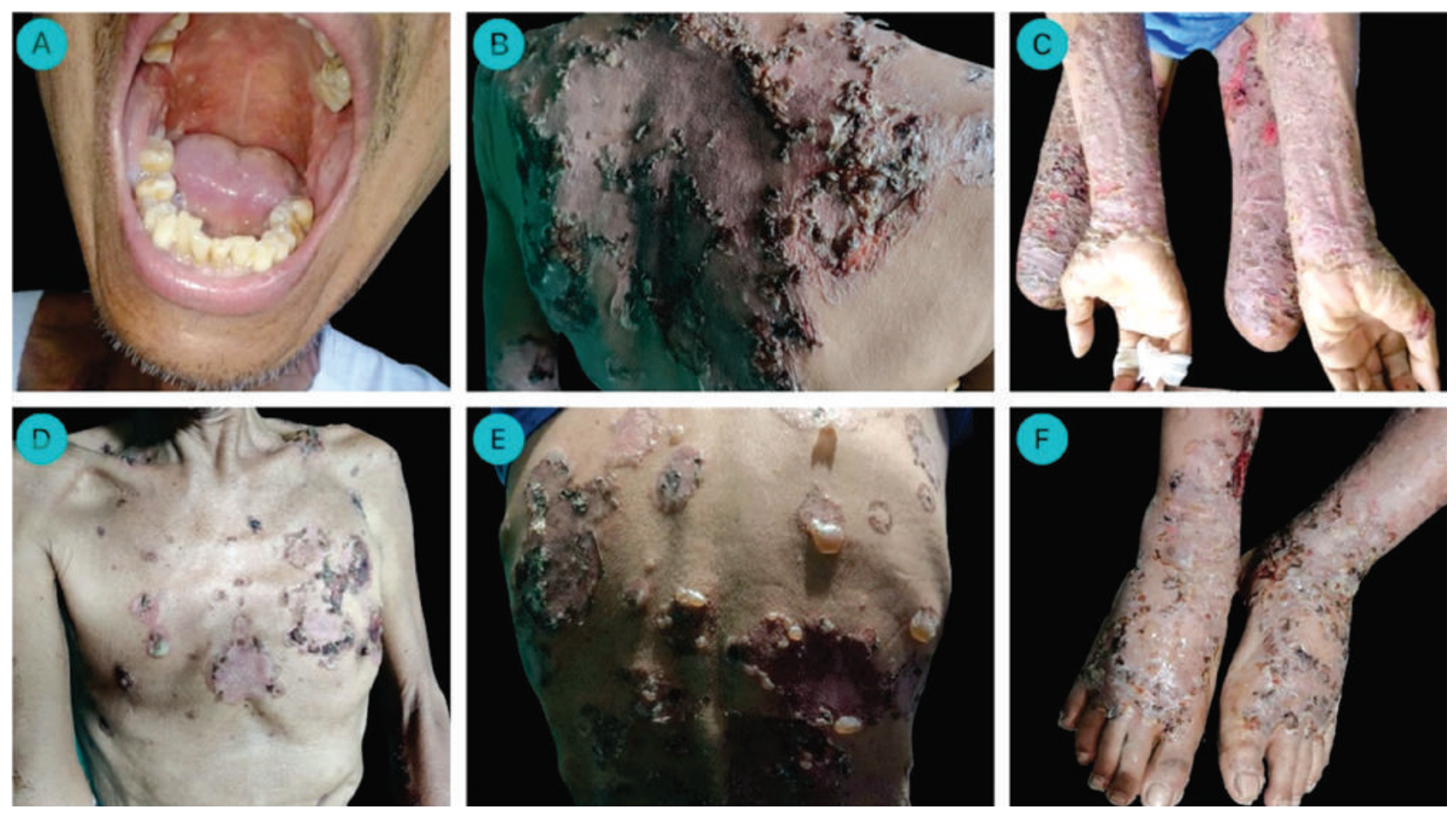

Figure 1. Photos of the patient during the initial telemedicine consult. Multiple, tense vesicles and bullae on erythematous base, some were eroded and crusted, forming an annular configuration on the trunk and extremities (B, C, D, E, F). No mucosal lesions were appreciated (A). 

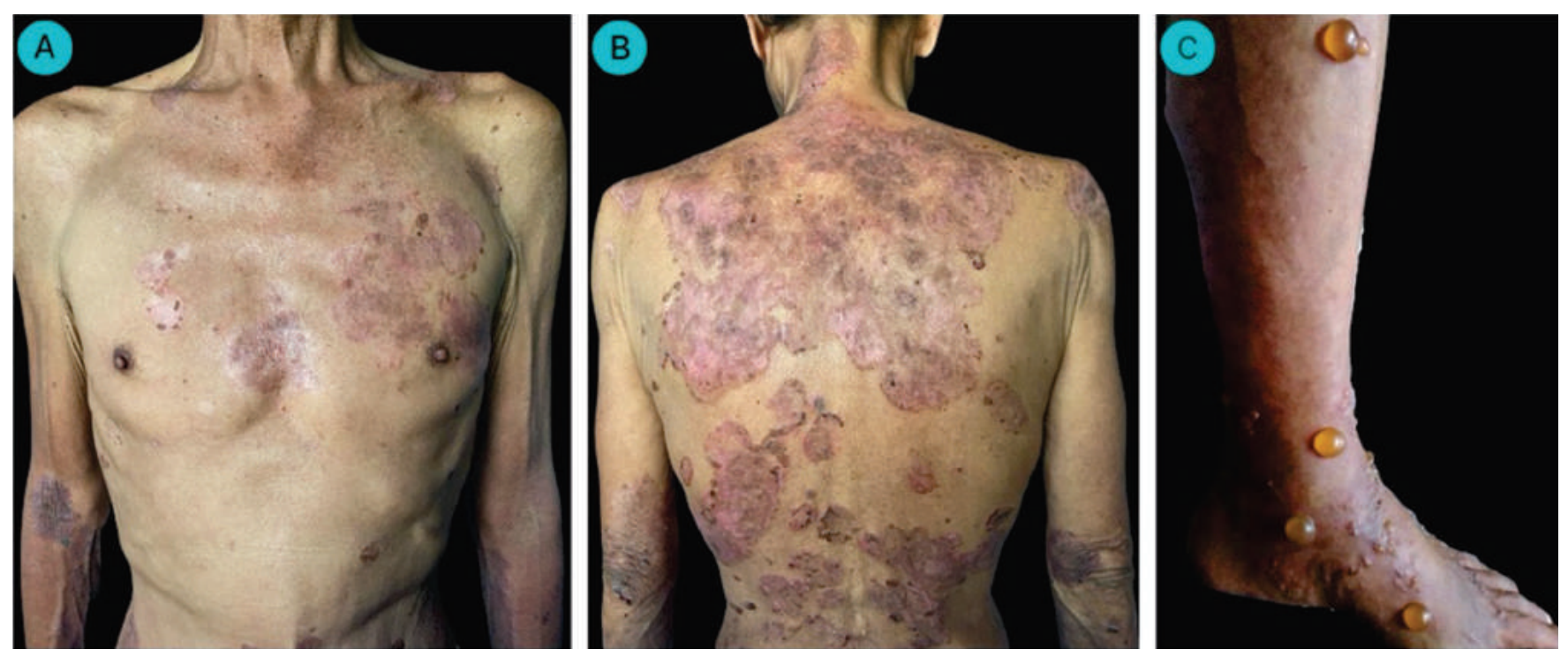

Figure 2. Marked improvement after more than a week of high dose prednisone was observed (A, B). New tense bullae and vesicles containing yellow-orange serous fluid appeared and were most prominent on the lower extremities (C).
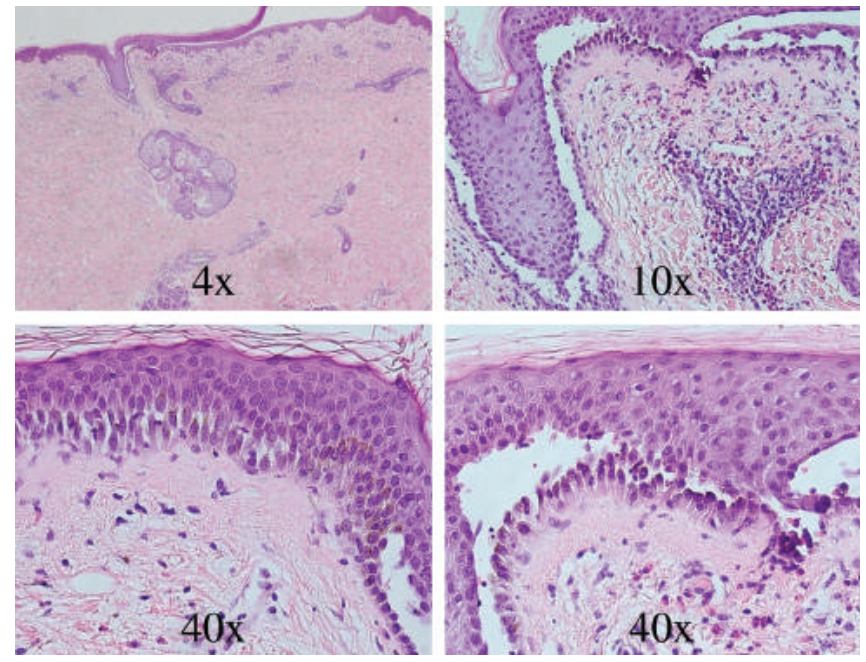

Figure 3. Histopathologic review of 4-mm punch biopsy taken from the edge of an intact bulla. Sections show intraepithelial splitting, suprabasal acantholysis, and tombstoning pattern.

basket-weave orthokeratosis, suprabasal acantholysis, and eosinophilic spongiosis were appreciated. In the dermis, moderate superficial perivascular and interstitial inflammatory infiltrates composed of neutrophils, eosinophils, and lymphocytes were seen. (Figure 3).

Direct immunofluorescence of perilesional skin showed faintly positive intercellular deposition of immunoglobulin $\mathrm{G}(\mathrm{IgG})$ and intensely positive intercellular deposition of complement $3(\mathrm{C} 3)$ predominantly on the lower spinous and basal layer resembling a chicken coop wire mesh (Figure 4).

These findings confirmed pemphigus vulgaris. Prednisone $(0.5 \mathrm{mg} / \mathrm{kg} / \mathrm{day})$ was continued, and mycophenolate mofetil $(30 \mathrm{mg} / \mathrm{kg} /$ day) in two divided doses was prescribed.
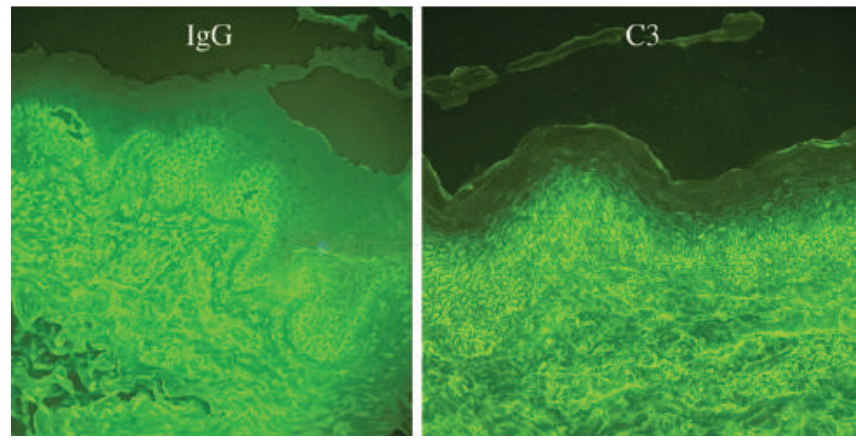

Figure 4. Direct immunofluorescence of perilesional skin. Sections show intercellular deposition of $\lg$ (+) and $\mathrm{C} 3(++)$ predominantly in the lower spinous and basal layer.

The latter was intended to become his maintenance steroidsparing medication as the plan was to slowly taper him off prednisone. However, because the patient could not afford mycophenolate mofetil, the authors gave him a free supply of dapsone $(50 \mathrm{mg}$ once daily) during the remission induction phase of the disease and also as monotherapy during the maintenance phase once he is off prednisone. ${ }^{4,5}$

More than two months after commencing treatment, the patient's DLQI score improved from 25 to 7 (dermatosis resulting in a moderate adverse effect on the patient's life). On follow-up by telemedicine, resolution of all his previous lesions was observed, and no new blisters were appreciated (Figure 5).

Aside from DLQI score improvement, the more specific quality of life measures validated for patients with autoimmune bullous disease, particularly pemphigus vulgaris, also improved - his Pemphigus Disease Area Index (PDAI) and Autoimmune Bullous Disease Quality of Life (ABQOL) 

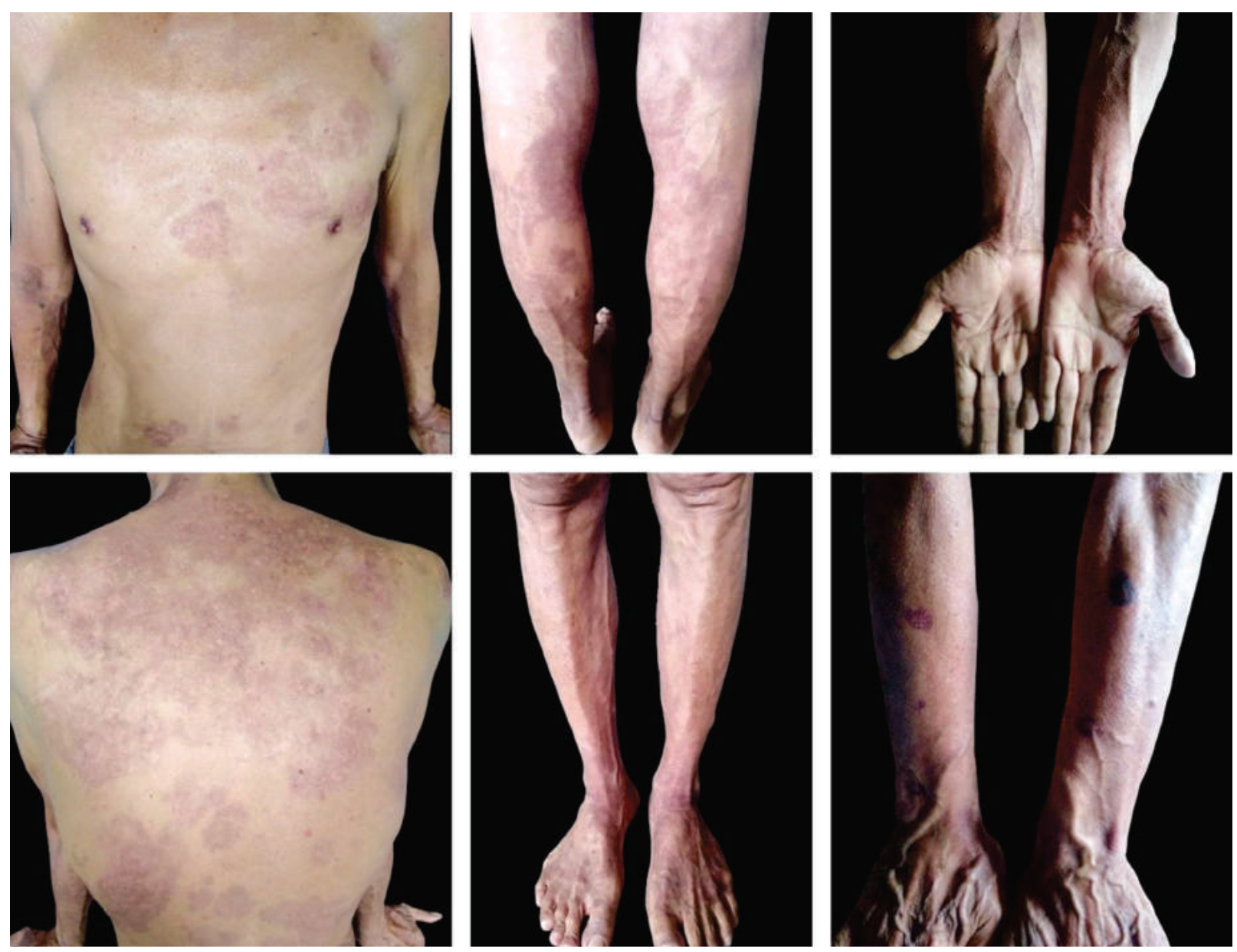

Figure 5. Completeresolution of lesions aftertwo months of therapy. Well-demarcated, irregularly shaped, hyperpigmented patches with scalloped borders and paler center are seen on sites of previous vesicles and bullae.

scores improved from 60 to 8 and 38 to 13 , respectively., 6,7 With improvement in quality of life, the patient regained prior functionality to perform activities of daily living.

\section{DISCUSSION}

Pemphigus vulgaris belongs to a spectrum of rare autoimmune mucocutaneous bullous dermatoses. ${ }^{8}$ It is characterized by flaccid blisters and erosions of the skin and/or mucous membranes with a progressive course that is likely fatal if left untreated. ${ }^{9}$ The clinical appearance of flaccid blisters in pemphigus results from the production of autoantibodies that target keratinocyte adhesion proteins, particularly desmogleins 1 and 3, leading to the loss of keratinocyte adhesion or acantholysis. In PV, acantholysis occurs just superior to the epidermis' basal layer, leading to painful flaccid blisters. ${ }^{10}$

The presentation of tense vesicles and bullae in our patient with PV was peculiar but not unheard of. Yoshida et al. first described a case of a 57-year-old Japanese female who presented with a 6-month history of annular erythematous lesions with papules, vesicles, tense bullae, and crusted erosions on the trunk and extremities, with no associated mucosal involvement. ${ }^{11}$ Initial diagnosis was linear IgA bullous dermatosis (LABD) or vesicular pemphigoid, but histopathology and DIF studies showed features that were compatible with PV similar to our patient.

The second case was reported by Nguyen et al. in a 51-year-old female with diabetes mellitus type 2 and dyslipidemia who presented with tense vesicles and bullae on an erythematous base, some also in annular configuration. Similar to our patient, biopsy showed suprabasal acantholysis, and DIF studies showed characteristic intercellular IgG and $\mathrm{C} 3$ deposition consistent with PV. ${ }^{12}$

Shinkuma et al. reported the third case of pemphigus vulgaris with both tense and flaccid blisters on the trunk and extremities and none on any mucosal surface in a 50year old male. The histopathology and DIF studies were also similar with our case and with those of Yoshida et al. and Nguyen et al. ${ }^{13}$

None of these earlier reports offered a probable explanation for this unusual presentation of PV. Shinkuma et al. explored the two common subtypes of PV - mucosal dominant type (mPV) and the mucocutaneous type (mcPV) 
Pemphigus Vulgaris with Tense Blisters in a 58-year-old Filipino Male

- and a rare third subtype called cutaneous type (cPV) that has variable presentation. Cutaneous PV may show features similar to pemphigus foliaceous (PF), vesicular pemphigoid, seborrheic dermatitis, discoid lupus erythematosus, eczema, and linear IgA bullous dermatosis (LABD) as in the case of our patient. ${ }^{13}$ The desmoglein compensation theory, which explains why oral lesions occur in PV but not in $\mathrm{PF}$, offers a clear explanation of why $\mathrm{PF}, \mathrm{mPV}$, and $\mathrm{mcPV}$ present the way they do clinically. However, this theory does not explain the variable presentation of $\mathrm{cPV}$, particularly the appearance of tense blisters similar to subepidermal blistering diseases like LABD, in rare cases of PV.

With the advent of protein array technology, new studies have been able to identify new antigen targets of PV autoantibodies aside from desmogleins 1 and 3. This includes calcium ATPase pump (implicated in Hailey-Hailey disease, which presents with acantholysis at the same level as PV), and human leukocyte antigen (HLA) proteins (implicated in pemphigoid gestationis), among others. ${ }^{10}$

To explain the variable presentation of $\mathrm{cPV}$, the "supercompensation hypothesis" has been proposed considering the newly identified antigens that are hypothesized to be targeted by PV autoantibodies aside from desmogleins. ${ }^{10}$

Plans for this patient include using the BIOCHIP mosaic-based indirect immunofluorescence technique further to characterize the existing autoantibodies in the patient's serum.

\section{CONCLUSION}

Our current understanding of autoimmune bullous dermatosis is constantly evolving. To our knowledge, there have only been three published cases of pemphigus vulgaris presenting as tense blisters. In addition, new hypotheses are being explored to explain the pathophysiology of this complex group of blistering skin diseases. The exact mechanism that leads to the clinical presentation of tense blisters in this rare clinical phenotype of PV needs elucidation. With a better understanding of their exact pathologic mechanisms, new therapeutic targets may be identified, offering a better quality of life to PV patients.

Pemphigus vulgaris requires close follow-up with a dermatologist, especially during the initial phase of treatment when a systemic corticosteroid has to be slowly tapered to the lowest dose that can achieve disease control while waiting for a steroid-sparing medication like MMF to exert its effect.

The quarantine restrictions brought about by the current COVID-19 pandemic delay access to a specialist leading to incorrect diagnosis and delayed treatment, especially in atypical cases such as our case.

While PV treatment remains challenging regardless of phenotype due to the corticosteroid-dependent nature of PV and the adverse effects of chronic immunosuppression, dermatologists may take advantage of telemedicine to manage $\mathrm{PV}$ patients as pemphigus is a notoriously challenging disorder to treat, and even more so during the time of COVID-19.

\section{Statement of Authorship}

Both authors participated in data collection and analysis, and approved the final version submitted.

\section{Author Disclosure}

Both authors declared no conflicts of interest.

\section{Funding Source}

No funding support.

\section{REFERENCES}

1. Kang S, Amagai M, Bruckney AL, Enk AH, Margolis DJ, McMichael AJ, Orringer JS. Chapter 15 Epidermal and Dermal Adhesion. In S Kang et al (eds). Fitzpatrick's Dermatology, 9e. 2019 (pp. 232-248). McGraw-Hill Education.

2. Finlay AY and Khan GK. Dermatology Life Quality Index (DLQI): A Simple, Practical Measure for Routine Clinical Use. Clin Exp Dermatol 1994; 19:210-216. doi: 10.1111/j.1365-2230.1994.tb01167.x.

3. Nicholas MW, Rao CL, Hall RP. Chapter 58 Linear Immunoglobulin A Dermatosis and Chronic Bullous Disease of Childhood. In S Kang et al (eds). Fitzpatrick's Dermatology, 9e. 2019 (pp. 992-1001). McGraw-Hill Education.

4. Baum S, Debby A, Gilboa S, Trau H, Barzilai A. Efficacy of Dapsone in the Treatment of Pemphigus Vulgaris: A Single-Center Case Study. Dermatology 2016; 232(5):578-585. doi: 10.1159/000448028.

5. Werth VP, Fivenson D, Pandya AG, (max of 6 authors), et al. Multicenter Randomized, Double-blind, Placebo-controlled, Clinical Trial of Dapsone as a Glucocorticoid-sparing Agent in Maintenancephase Pemphigus Vulgaris. Arch Dermatol 2008; 144:25-32.

6. Rosenbach M, Murrell DF, Bystryn JC, Dulay S, Dick S, Fakharzadeh S, Hall R, Korman NJ, Lin J, Okawa J, Pandya AG, Payne AS, Rose M, Rubenstein D, Woodley D, Vittorio C, Werth BB, Williams EA, Taylor L, Troxel AB, Werth VP. Reliability and Convergent Validity of Two Outcome Instruments for Pemphigus. J Invest Dermatol 2009 Oct; 129(10):2404-10. doi: 10.1038/jid.2009.72.

7. Sebaratnam DF, Hanna AM, Chee SN, Frew JW, Venugopal SS, Daniel BS, Martin LK, Rhodes LM, Tan JC, Wang CQ, Welsh B, Nijsten T, Murrell DF. Development of a Quality-of-life Instrument for Autoimmune Bullous Disease: The Autoimmune Bullous Disease Quality of Life Questionnaire. JAMA Dermatol 2013 Oct; 149(10):1186-91. doi: 10.1001/jamadermatol.2013.4972.

8. Murrell DF, Peña S, Joly P, Marinovic B, Hashimoto T, Diaz L. et al. Diagnosis and Management of Pemphigus: Recommendations of an International Panel of Experts. J Am Acad Dermatol 2020 Mar; 82(3): 575-585.e1. doi: 10.1016/j.jaad.2018.02.021.

9. Bilgic A, Murrell DF. What is Novel in the Clinical Management of Pemphigus? Expert Rev Clin Pharmacol 2019 Sep; 12(10): 973-980. doi: 10.1080/17512433.2019.1670059.

10. Sinha AA, Sajda T.The Evolving Story of Autoantibodies in Pemphigus Vulgasris: Development of the "Super Compensation Hypothesis". Front Med (Lausanne) 2019 Aug; 5:218. doi: 10.3389/fmed.2018.00218

11. Yoshida K, Takae Y, Saito H, Oka H, Tanikawa A, Amagai M., et al. Cutaneous Type Pemphigus Vulgaris: A rare Clinical Phenotype of Pemphigus. J Am Acad Dermatol 2005 May; 52:839-845. doi:10.1016/j.jaad.2005.01.106.

12. Nguyen ET, Lin SK, Wu JJ.Pemphigus Vulgaris with Tense Bullae. Perm J 2015 Winter; 19(1): 77-78. http://dx.doi.org/10.7812/TPP/14-131.

13. Shinkuma S, Nishie W, Shibaki A, Sawamura D, Ito K, Tsuji-Abe Y. et al. Cutaneous Pemphigus Vulgaris with Skin Features Similar to the Classic Mucocutaneous Type: A Case Report and Review of Literature. Clin Exp Dermatol 2008 Nov; 33(6):724-728. doi: 10.1111/j.1365-2230.2008.02871.x. 